\title{
Control interno y gestión financiera de una empresa proveedora de alimentos
}

Internal control and financial management of a food supplier company

Controle interno e gestão financeira de uma empresa fornecedora de alimentos

\author{
Avelino Villafuerte de la Cruz \\ avillafuerte@ucv.edu.pe \\ Universidad César Vallejo, Tarapoto, Perú \\ ORCID 0000-0002-9447-8683 \\ Segundo Eloy Soto Abanto \\ ssotoab@ucvvirtual.edu.pe \\ Universidad César Vallejo, Trujillo, Perú \\ ORCID 0000-0003-1004-5520 \\ Nury Acosta Ruiz \\ aaostaru@ucvvirtual.edu.pe \\ Universidad César Vallejo. Tarapoto, Perú \\ ORCID 0000-0002-0529-8630 \\ Herminia Liry Chavez Huashuayllo \\ hchavezhua@ucvvirtual.edu.pe \\ Universidad César Vallejo, Lima Norte, Perú \\ ORCID 0000-0003-3165-8289
}

\section{RESUMEN}

Objetivo: determinar el efecto del control interno en la gestión financiera de una empresa proveedora de alimentos en el 2020. Metodología: El diseño de la investigação cuantitativa, de diseño no experimental, de corte transversal; la técnica utilizada para la recolección de datos fue la entrevista, observación y análisis documental. Resultados: determinaron que, la empresa proveedora de alimentos presenta deficiencias en los componentes de control interno; donde se llegó a evidenciar faltante de existencias, deterioro, mercadería extraviada y vencimiento sumando un total de S/ 48,296.90 en pérdidas. Asimismo, después de haber efectuado el ajuste de los hallazgos se determinó que la empresa hubiera obtenido un mayor rendimiento, es decir los indicadores de liquidez y rentabilidad hubieran sido superior a lo obtenido. Conclusión: el control interno incide en la gestión financiera de la empresa proveedora de alimentos.

Palabras clave: control, gestión, monitoreo, rentabilidad.

\begin{abstract}
Objective determine the effect of internal control on the financial management of a food supplier company in 2020. Methodology: The data collection technique was the interview, observation and documentary analysis. The techniques corresponded to the interview guide, verification sheet and document analysis guide. Results: determined that the food supplier company has deficiencies in the internal control components; where there was evidence of a shortage of stocks, deterioration, lost merchandise and expiration, adding a total of S / 48,296.90 in losses. Likewise, after having adjusted the findings, it was determined that the company would have obtained a higher return, that is, the liquidity and profitability indicators would have been higher than what was obtained Conclusion: that internal control affects the financial management of the food supplier company.
\end{abstract}

Keywords: control, management, monitoring, profitability. 


\section{INTRODUCCIÓN}

El control interno es una herramienta fundamental para las organizaciones, pues permite comprender la situación real de las empresas por medio de actividades de medición, gestión y planificación, en relación con aspectos como la efectividad y eficiencia de las operaciones, confiabilidad de los informes financieros o el acatamiento de reglas y normas establecidas, para garantizar principalmente el cumplimiento de los objetivos empresariales (Vivanco, 2017).

Desde el ámbito internacional, Sanusi et al., (2015) fundamentan que desde hace muchos años una gran variedad de empresas que se dedican a la venta de bienes y servicios enfrentan un problema en común, que es la falta de fondos para desempeñar sus actividades de forma oportuna debido al deficiente manejo de efectivo, pues las diferentes situaciones económicas por las que atraviesan los países no solo afecta el nivel de demanda de los productos o servicios de las organizaciones, sino que también ponen trabas al correcto manejo del cobro de sus cuentas por cobrar.

Este problema en la empresa proveedora de alimentos se manifiestan negativamente en sus actividades empresariales como en el pago tardío de los trabajadores y proveedores, disminución de los ingresos, falta de liquidez y baja rentabilidad, inconvenientes en el stock del almacén, vencimiento de los productos perecibles, entrega a destiempo de sus productos lo que ocasiona disgustos e insatisfacción por parte de los clientes. Asimismo, otros de los efectos negativos exteriorizados en la empresa por las deficiencias en el control interno y la gestión financiera, son la falta de información veraz y actualizada para la toma de decisiones, incremento de sus deudas por pagar, problemas para acceder a financiamiento, fallas en la protección de los activos. Debido a todo lo expuesto, la liquidez y rentabilidad de la empresa se ha visto afectada en diferentes aspectos, por lo que nace el interés de realizar el estudio del control interno y la gestión financiera de la empresa proveedora de alimentos.

Para estudiar el efecto del control interno en la gestión financiera de la empresa proveedora se consideró los aportes de ciertos autores para la evaluación de cada variable. En cuanto al control interno, su evaluación estuvo fundamentada en la teoría de Pereira (2019) quien dimensiona al control interno en cinco componentes (ambiente de control, evaluación de riesgos, actividades de control, sistemas de información y comunicación, supervisión y monitoreo). En el caso de la gestión financiera, se tomó en cuenta lo manifestado por Apaza y Barrantes (2020) quienes señalan que su evaluación se realiza por medio de ratios de liquidez y rentabilidad.

En efecto, por la problemática descrita se formuló el siguiente problema general: ¿cuál es el efecto del control interno en la gestión financiera de una empresa proveedora de alimentos en el 2020? y como problemas específicos: ¿cómo es el desempeño de las actividades de control interno de una empresa proveedora de alimentos en el 2020?; ¿cuáles son las deficiencias de las actividades de control interno de una empresa proveedora de alimentos en el 2020?; y ¿cuáles son los índices de la gestión financiera de una empresa proveedora de alimentos en el 2020?

\section{FUNDAMENTO TEÓRICO}

\subsection{Antecedentes}

\subsection{Teorias relacionadas (Variables, dimensiones)}

Desde el ámbito internacional, Sotomayor et al., (2020) en su artículo científico desarrollado en la ciudad de Machala - Ecuador, establecieron como objetivo determinar la forma en que el control 
interno contribuye a una mejor gestión contable y financiera de las empresas camaroneras de la ciudad de Machala. La investigación fue desarrollada bajo un enfoque cualitativo, con diseño documental, bibliográfico y descriptivo. Como técnicas de recolección de datos se aplicó el análisis documental y la encuesta, y los instrumentos utilizados fueron las fichas bibliográficas y el cuestionario. La muestra estuvo compuesta por 8 empresas camaroneras. En cuanto a los resultados, la característica de mayor relevancia que debe tener un adecuado control interno según las empresas camaroneras $(50 \%)$ es de tipo normativo, puesto que esta ayuda a evitar conflictos con los organismos encargados de control. Además, el 50\% de los encuestados consideró que el sistema contable de la empresa camaronera se debió evaluar a través de aspectos reglamentarios. De igual manera un $24 \%$ afirmó que la técnica contable tiene que ser considerada para evaluar el control interno. Asimismo, el $62.5 \%$ creen que llevar un adecuado control interno contribuye al crecimiento de las utilidades y del desempeño organizacional. Concluyeron que control interno es una herramienta fundamental para las empresas camaroneras, ya que les permite llevar un mejor manejo $\mathrm{y}$ tener un control total de sus recursos humanos, financieros y materiales. Del mismo modo, permite a los directivos de la organización obtener una visión amplia e integral acerca de la situación de la organización, contando con información real de los activos.

A continuación, se muestra la fundamentación teórica de las variables en estudio. El control interno, según Pereira (2019) es un proceso ejecutado por los miembros de una empresa con el fin de obtener un control apropiado respecto al desempeño y desarrollo de actividades que se deben realizar para que la organización logre el cumplimiento de sus objetivos. Asimismo, Meléndez (2016) refiere que el control interno es planteado con el fin de garantizar el logro de los objetivos de un organismo empresarial, buscando que las operaciones sean efectivas y eficientes, resaltando que es un proceso en el que es indispensable la participación de los directivos, gerentes, colaboradores y la red de procesos de una organización. Por su parte, Pelazas (2015) lo define como mecanismos y técnicas que son aplicados en los procesos de planeación y gestión, para la toma de decisiones y para direccionar el logro y cumplimiento de las metas.

La segunda variable es la gestión financiera, considerada por Fajardo y Soto (2018) como un procedimiento administrativo cuyo propósito es analizar, evaluar, obtener y utilizar los recursos financieros de una determinada empresa. Por su lado, Apaza y Barrantes (2020) manifiestan que la gestión o administración financiera hace referencia a un conjunto de diversas actividades que son ejecutadas para alcanzar el cumplimiento eficiente de los objetivos y garantizar la rentabilidad, solvencia, liquidez y un buen desenvolvimiento de las empresas. Asimismo, Armengol (2017), la define como una disciplina, encargada de analizar las acciones y decisiones relacionadas con los medios económicos-financieros necesarios en el desarrollo de las operaciones de las organizaciones, por medio de los cuales se busca elevar el nivel del valor de la empresa.

\section{PROCEDIMIENTOS METODOLÓGICOS}

Tomando como referencia la definición de CONCYTEC (2018), la investigación fue de tipo aplicada, puesto que con el desarrollo del estudio y tras un análisis previo de las teorías se pudo evaluar el comportamiento del control interno y la gestión financiera, con el fin de establecer acciones de mejora frente a las deficiencias encontradas en la empresa proveedora.

Acorde a la metodología el estudio contó con un enfoque cuantitativo y tuvo un diseño no experimental, ya que las variables (control interno y gestión financiera) no fueron manipuladas y evaluadas, esto quiere decir que se presentaron tal cual. Asimismo, fue de corte transversal dado que la información y los datos necesarios para la investigación fueron recolectados de un periodo en específico. 
También es preciso mencionar que la investigación tuvo un alcance descriptivo, ya que se expuso las características y propiedades de las variables con la finalidad de determinar el efecto del control interno en la gestión financiera.

Se redactó un documento solicitando la autorización correspondiente al gerente general de la empresa con la finalidad de realizar la investigación con las facilidades que correspondan. En cuanto a la recopilación de datos, se aplicó una entrevista al administrador de la empresa proveedora de alimentos y también se solicitó información concerniente a la gestión financiera al jefe de contabilidad de la organización. Luego, se realizó la observación del control interno dentro de la organización para determinar las deficiencias de las mismas. Por consiguiente, los datos recopilados fueron tabulados y posteriormente plasmados en Excel para ser mostrados en el trabajo de investigación.

En cuanto al método de análisis de datos, se aplicó el método descriptivo, pues estuvo basado en el estudio de las distribuciones de una variable, de manera que se pueda obtener un resultado por medio de los elementos de la muestra, en este caso la empresa proveedora en el 2020. Para ello, se emplearon recursos estadísticos que permitieron explicar de forma coherente los resultados, así como su comparación con otros periodos con la finalidad de explicar el efecto del control interno en la gestión financiera. Por otra parte, en cuanto a la gestión financiera se realizó el análisis vertical y horizontal en base a los Estados Financieros (Estado de situación financiera y Estado Integral), y para brindar mayor confiabilidad y reconocimiento se aplicaron los indicadores de rentabilidad y liquidez para medir el rendimiento. La empresa proveedora de alimentos se encuentra en la región de loreto en Alto Amazonas ubicado en el distrito de Yurimagua.

\section{RESULTADOS Y DISCUSIÓN}

Seguidamente para dar respuesta a este capítulo se realizó el trabajo de campo sobre la variable control interno en una empresa proveedora de alimentos. Asimismo, se analizó toda la información recopilada, el cual permitió evaluar el desempeño de las actividades del control interno como primer objetivo específico:

Evaluar el desempeño de las actividades de control interno de una empresa proveedora de alimentos en el 2020.

\section{Tabla 2}

Cumplimiento de actividades dimensión ambiente de control

Dimensión Actividades Cumplimiento

Las actividades del negocio están estructuradas de forma adecuada.

Ambiente de Se asigna anualmente a las autoridades y se planifican las control actividades de control a realizar.

No

Se difunde y se promueve el cumplimiento de los valores y normas establecidas por empresa.

\section{Interpretación:}


De acuerdo a la presente tabla, se observa claramente que la empresa proveedora de alimentos incurre a un bajo cumplimiento de actividades en la dimensión ambiente de control, esto indica que, no se asignan responsabilidades y sobre todo no se planifican las tareas operativas dentro del negocio. Por otra parte, se logró evidenciar que no se promueve y difunden la ejecución de normas y valores que el personal encargado debe integrar dentro de sus labores, pues esto viene limitando su función dentro de la misma haciendo que los resultados cualitativos y cuantitativos se resistan a un cambio positivo. En síntesis, se comprobó que la empresa a nivel de gestión no adapta las estrategias y herramientas que mantiene para generar un cambio favorable en su manual de funciones, esto hace que el ambiente de control no concatene indicadores positivos que sumen a la empresa y le otorgue mayores oportunidades de mejora en sus procedimientos, pues habitualmente la organización suele utilizar los recursos que se encuentran disponibles, esto indica que la gerencia no estima un presupuesto para la adquisición de nuevas herramientas que permitan valorar los inventarios aumentando el margen de pérdidas al realizar la verificación física de cada uno de estos.

\section{Tabla 3}

Cumplimiento de actividades dimensión evaluación de riesgos

\begin{tabular}{clc}
\hline \multicolumn{1}{c}{ Dimensión } & \multicolumn{1}{c}{ Actividades } & Cumplimiento \\
\hline & $\begin{array}{l}\text { La evaluación de los efectos de los riesgos se realiza } \\
\text { continuamente. }\end{array}$ & $\mathrm{Si}$ \\
Evaluación de riesgos & $\begin{array}{l}\text { Se determinan acciones y controles para enfrentar } \\
\text { posibles riesgos. } \\
\text { Existe una evaluación periódica del cumplimiento de } \\
\text { los procesos. }\end{array}$ & No \\
\hline
\end{tabular}

\section{Interpretación:}

En relación a la presente tabla, se identificó que la empresa registra un bajo cumplimiento de actividades en el componente evaluación de riesgos, esto resuelve que, no se determinan efectivamente acciones y un control para poder enfrentar los problemas que se producen en la adquisición de las mercaderías que suelen solicitarse de manera frecuente, pues esto suma a la limitada evaluación periódica del responsable de área y el bajo cumplimiento de procesos que se desarrollan internamente. En términos numérico de cumplimiento se puede observar que, 2 de las 3 actividades no se cumplen de manera oportuna generando problemas los procesos logísticos.

Por otra parte, en relación a los aspectos que asume la organización en el control de riesgo ello no se orienta a la valoración, análisis y fraudes trayendo un impacto negativo a nivel de gestión. Esto hace entender que la evaluación de riesgo no se desarrolla de manera correcta haciendo que genere contingencias y deficiencias internas.

\section{Tabla 4}

Cumplimiento de actividades dimensión actividades de control

Dimensión

Actividades de

Actividades

Se resguarda y preserva correctamente los activos y

bienes de la organización
Cumplimiento

No 
control

Se analiza constantemente los registros de la empresa que contienen información relevante.

Se toma en cuenta la revisión de informes para la toma de decisiones.

\section{Interpretación:}

Con respecto a la presente tabla, se observa que la empresa proveedora de alimentos no cumple totalmente con las actividades de control que se producen de manera interna, esto indica que, no que existe un resguardo y preservación de los activos y bienes que se tienen en los registros contables, pues esto viene reduciendo la toma de decisiones a fin de poder obtener un mayor rendimiento en la empresa considerándose el rubro en que se encuentra.

Además, no mantiene actualizaciones de seguridad, lo cual dificulta establecer una correcta programación donde las actividades que se desarrollen internamente no se vean interrumpidas y las entregas puedan darse sin mayor dificultad.

\section{Tabla 5}

Cumplimiento de actividades dimensión información y comunicación

Dimensión

Actividades

Los resultados obtenidos son comunicados a

los miembros de la empresa.

Sistemas de información

y comunicación
Cumplimiento

No

$\mathrm{Si}$

\section{Interpretación:}

De acuerdo a los resultados de la presente tabla, se observa claramente que las actividades del sistema de información y comunicación no se cumplen en su totalidad, esto demuestra que la empresa no mantiene información actualizada de sus productos siendo notable en el proceso de distribución reduciendo las ventas por la falta de disponibilidad limitando de manera notable las actividades operativas y comerciales como empresa proveedora. La comunicación es muy reducida entre el responsable de área y gerencia influyendo negativamente en la toma de decisiones. En cuanto a la verificación de los resultados, ello no suele realizarse según los reportes y saldos que se registran en el sistema.

\section{Tabla 6}

Cumplimiento de actividades dimensión supervisión y monitoreo

Dimensión Actividades Cumplimiento

\begin{tabular}{lll}
\hline $\begin{array}{l}\text { Supervisión y } \\
\text { monitoreo }\end{array}$ & $\begin{array}{l}\text { Se realiza un seguimiento apropiado de las } \\
\text { cuentas bancarias de la empresa. }\end{array}$ & No \\
& Se actualiza periódicamente los datos y la & No
\end{tabular}


información concerniente a la empresa.

\section{Interpretación:}

Como se puede observar en la presente tabla, la empresa proveedora de alimentos no cumplen con lo que se estipula dentro de las actividades de supervisión y monitoreo, esto refiere que, no se brinda el seguimiento frecuente de las cuentas bancarias que maneja la organización, además, no se actualiza frecuentemente la información concerniente a la empresa de sus inventarios, esto indica que la empresa no tiene una fecha específica donde se pueda verificar la consistencia, estado e integridad 


\section{Tabla 7}

Análisis de ratios de liquidez

\begin{tabular}{lccc}
\hline \multicolumn{1}{c}{ Ratios } & $2019(\%)$ & $2020(\%)$ & $\begin{array}{c}\text { Variación } \\
(\%)\end{array}$ \\
\hline Ratio de solvencia & 2.06 & 2.01 & -0.05 \\
Prueba ácida & 3.32 & 1.48 & -1.83 \\
Capital de trabajo & S/ 4,109,168.54 & S/ 4,407,835.30 & S/ 298,666.76 \\
\hline
\end{tabular}

\section{Interpretación:}

Como se observar en la presente tabla, la empresa proveedora por cada sol de pasivo corriente posee un respaldo de 2.06 y 2.01 en el activo circulante para responder sus obligaciones a corto plazo con terceros en el año 2019 y 2020 respectivamente, asimismo, ello refleja una variación con tendencia negativa afirmándose de esta forma que la empresa registra una limitación moderada en relación a capacidad de pago. En cuanto a la prueba ácida, este indicador es similar al ratio de solvencia, indicando que la empresa proveedora tiene un mayor problema de liquidez sin considerar los activos realizables que serían las existencias. Para finalizar el capital de trabajo, el cual comprende el saldo económico que le queda a la organización después de deducir los pasivos circulantes, llegó a registrar un indicador valor de, S/ 4,109,168.54 S/ 4,407,835.30 el mismo que no fue esperado reduciendo las inversiones que se producen de manera frecuente a corto y largo plazo.

\section{Tabla 8}

Análisis de ratios de rentabilidad

\begin{tabular}{lccc}
\hline \multicolumn{1}{c}{ Ratios } & 2019 & 2020 & Variación \\
\hline Rentabilidad sobre el patrimonio & $5.43 \%$ & $4.96 \%$ & $-0.47 \%$ \\
Retorno del capital empleado & $54.80 \%$ & $26.52 \%$ & $-28.29 \%$ \\
Rendimiento sobre los activos & $3.02 \%$ & $2.69 \%$ & $-0.33 \%$ \\
Rentabilidad neta & $0.78 \%$ & $0.85 \%$ & $0.06 \%$ \\
Margen de utilidad bruta & $7.58 \%$ & $9.21 \%$ & $1.63 \%$ \\
Margen de utilidad operativa & $1.18 \%$ & $1.31 \%$ & $0.13 \%$ \\
\hline
\end{tabular}

\section{Interpretación:}

Según como se observa en la tabla, la empresa proveedora reflejó una variación notable en relación a los indicadores de rentabilidad, dando así a conocer en primera instancia que el retorno de capital empleado fue bajo $54.80 \%$ (2019) y $26.52 \%$ (2020) poniendo en manifiesto que existe un deficiente manejo de recursos en la empresa que se contrasta con la baja gestión del gerente. Por otra parte, la rentabilidad sobre el patrimonio tuvo el mismo comportamiento con una variación de $5.43 \%$ a $4.96 \%$ en los últimos 2 años, reflejando que la empresa por cada sol de invertido obtuvo 0.543 y 
0.496 por los aportes accionistas. En cuando al rendimiento de activos totales, la empresa proveedora incurrió a una disminución de $3.02 \%$ a 2.69\%, indicando que la generación de utilidades a partir de la explotación de los activos. En síntesis, para finalizar el análisis correspondiente a la rentabilidad se afirma que la organización viene registrando problemas a nivel de gestión principalmente en la administración los activos más líquidos como son el efectivo, cuentas por cobrar comerciales, existencias.

\section{Tabla 09}

Incidencia de la gestión financiera con el control interno

\section{GESTIÓN FINANCIERA}

De las actividades deficientes que se desarrollaron en la empresa proveedora de alimentos, incurrieron a una pérdida por un monto de S/ 48,296.90, por la limitada revisión de los productos en el área de recepción, bajo resguardo y control de riesgos. Sin embargo, si las actividades se hubieran cumplido eficientemente la empresa hubiera registrado distintos resultados, como se detalla a continuación:

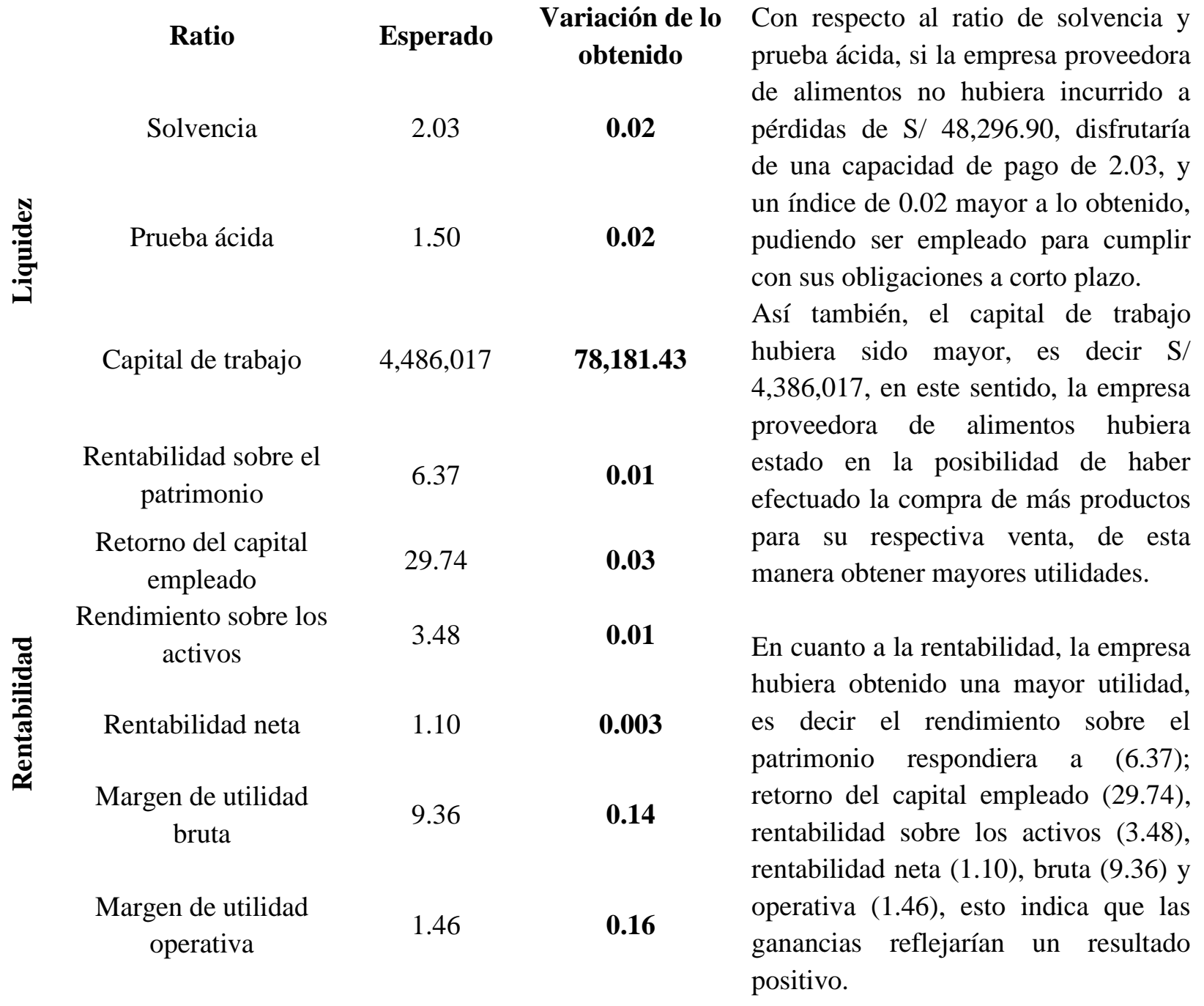

\section{EFECTO}

De esta manera en relación a los hallazgos se concluye que existe incidencia significativa entre las variables a estudiar.

Hi: El control interno tiene un efecto positivo en la gestión financiera de una empresa proveedora de alimentos en el 2020. 


\section{CONCLUSIÓN}

El control interno incide en la gestión financiera de la empresa proveedora de alimentos, motivo a que se presentaron un conjunto de actividades de forma incorrecta que incurrieron a pérdidas por un total de S/ 48,296.90, afectando directamente en el rendimiento como es la liquidez y rentabilidad de la empresa, sin embargo, sin la presencia de dichas deficiencias se hubiera obtenido un mayor resultado viéndose reflejado en la capacidad de pago y generación de utilidades.

En cuanto a la evaluación del desempeño del control interno en la empresa proveedora de alimentos, se conoció que no se cumplen efectivamente las actividades en el ambiente de control, evaluación de riesgos, actividades de control, información y comunicación, supervisión y monitoreo, esto indica que no son desarrolladas de forma correcta limitando la distribución de los productos y el desempeño de las funciones que se ejecutan de manera interna, lo cual que incide en la generación de beneficios a corto y largo plazo.

Se ha identificado las deficiencias en los componentes del control interno en la empresa proveedora de alimentos, siendo este la baja aplicación de instrumentos de control en el ingreso de los productos, incorrecto cotejo de la cantidad y calidad, el área de almacenamiento no determina las características necesarias para brindar un mayor resguardo a los productos de primera necesidad, falta de actualización del stock de los inventarios después de haber realizado el despacho respectivo (ventas), todo ello ha generado la pérdida de S/ 48,296.90

Al analizar la gestión financiera de la empresa proveedora de alimentos, se evidenció que incurrió a una variación en déficit donde la ratio de solvencia determinó en el primer año 2.06 y el segundo fue 2.01; esto resultado resuelve que la organización incurre a problemas en la capacidad de responder sus obligaciones a corto plazo. En relación a la rentabilidad determino una utilidad deficiente direccionando en primer lugar el Retorno del capital empleado con un coeficiente de $54.80 \%$ a $26.52 \%$, esto en síntesis indica que los indicadores no son satisfactorios para la gerencia para la ejecución de decisiones. En relación a la rentabilidad sobre el patrimonio determinó un resultado de $5.43 \%$ a $4.96 \%$. Sin embargo, solo el margen de utilidad bruta registró un índice positivo de $7.58 \%$ a $9.21 \%$. 


\section{REFERENCIAS}

Ackermann, C. (2017). A mixed methodology to view internal audits internal control functioning. Corporate Ownership and Control, 14(2), 302-311. https://doi.org/10.22495/cocv14i2c2p4

Ahmed, A., \& Muhammed, A. (2018). Internal control systems and its relationships with the financial performance in telecommunication companies "A case study of asiacell". Studies and scientific researches. economics edition, 7(28), 82-88. https://doi.org/10.29358/sceco.v0i28.416

Alva, E. (2017). La desaparición de las microempresas en el Perú. Una aproximación a los factores que predisponen a su mortalidad. Caso del Cercado de Lima. Economía y Desarrollo, 158(2), 76-90. https://www.redalyc.org/articulo.oa?id=425554493005

Apaza, M. \& Barrantes, E. (2020). Administración financiera. Pacifico Ediciones.

Arbaiza, L. (2014). Cómo elaborar una tesis de grado. ESAN Ediciones.

Armengol, P. (2017). Gestión de la inversión y el financiamiento. Herramientas para la toma de decisiones. Instituto Mexicano de Contadores Públicos, A.C. https://cutt.ly/Zmd098P

Baca, L. (2017). Implementación del control interno y su efecto en la gestión financiera de las agencias de viajes en el distrito de Miraflores, Lima [Tesis de pregrado, Universidad Autónoma del Perú]. https://cutt.ly/WnZFLQS

Bermúdez, H. (2016). Auditoría y control: Reflexiones a la luz de la legislación. Ediciones de la U. https://cutt.ly/MmfDOFb

Blanco, Y. (2015). Auditoría integral: normas y procedimientos. ECOE Ediciones. https://cutt.ly/JmfF1tr

Cabrera, C., Fuentes, M., \& Cerezo, G. (2017). La gestión financiera aplicada a las organizaciones. Dominio de las Ciencias, 3(4), 220-232. https://dialnet.unirioja.es/servlet/articulo?codigo $=6174482$

CONCYTEC (2018). Compendio de normas para trabajos escritos. ( $\left.1^{\circ} \mathrm{ed}\right)$. Colombia: Instituto Colombiano de Normas Técnicas y Certificación, CONCYTEC.

Estupiñán, R. (2015). Control interno y fraudes: análisis de informe COSO I, II y III con base en los ciclos transaccionales. ECOE Ediciones.

Fajardo, M. \& Soto, C. (2018). Gestión financiera empresarial. Ediciones UTMACH. http://repositorio.utmachala.edu.ec/bitstream/48000/14354/1/Cap.2\%20Fundamentos\%20de\%20la\%20gesti\% C3\%B3n\%20financiera.pdf

Ferrer, A., Apaza, M., Effio, F., García, J., Valdiviezo, J., Villanueva, S. \& Northcote, C. (2020). Planeamiento financiero en tiempos de pandemia del Covid-19. Pacifico Ediciones.

Flores, J. (2017). Finanzas aplicadas a la gestión empresarial. Centro de especialización en contabilidad y finanzas E.I.R.L

García-Moreno, E., Mapén-Franco, F., \& Berttolini-Díaz, G. (2019). Marco analítico de la gestión financiera en las pequeñas y medianas empresas: problemas y perspectivas de diagnóstico. CIENCIA UNEMI, 12(31), 128-139. https://doi.org/10.29076/issn.2528-7737vol12iss31.2019pp128-139p

Hernández-Sampieri, R. \& Mendoza, C. (2018). Metodología de la investigación. Las rutas cuantitativa, cualitativa y mixta. Editorial MCGraw Hill Education.

Homsi, M. (2017). The Internal Control in Financial Reporting. SSRN Electronic Journal, 1(1), 1-21. https://doi.org/10.2139/ssrn.3540049

Ibarra, D., Vega, L. y Carmenate, J. (2019) Auditoría y control interno en el control empresarial. Folletos Gerenciales, 23(2), 82-92. https://folletosgerenciales.mes.gob.cu/index.php/folletosgerenciales/article/view/205/214

Koo, J. \& Ki, E. (2020). Internal Control Personnel's Experience, Internal Control Weaknesses, and ESG Rating. Sustainability, 12(20), 2-16. https://doi.org/10.3390/su12208645

Lin, Z., Cao, R., Ding, M., \& Chang, Q. (2018). Internal Control Effectiveness, Relationship Trading and Corporate Reputation. Account and Financial Management Journal, 3(4), 1515-1529. https://doi.org/10.31142/afmj/v3i4.09

López, O. \& Guevara, J. (2016) Control Interno en Colombia: Un diagnóstico desde lo teórico. Revista de Investigación en Administración, Contabilidad, Economía y Sociedad, 4(5), 243-261. http://www.redalyc.org/articulo.oa?id=551857287010

Martínez, P. (2016). Control interno e integridad: elementos necesarios para la gobernanza pública. El Cotidiano, 18, 713. http://www.redalyc.org/articulo.oa?id=32546809002

Melendez, J. (2016). Control Interno. Universidad Católica Los Ángeles de Chimbote. https://cutt.ly/6nX3iVR

Nano, K. (2017). Caracterización del control interno y la gestión financiera de las micro y pequeñas empresas del sector comercio rubro ferreterías del distrito de Calleria, 2016 [Tesis de pregrado, Universidad Católica Los Ángeles Chimbote]. https://cutt.ly/QnZF9Cq

Ndegwa, R. \& Mungai, J. (2019). Influence of internal control system on financial management in ministry of finance, Kenya. International Academic Journal of Economics and Finance, 3(3), 266-278. https://iajournals.org/articles/iajef_v3_i3_266_278.pdf

Núñez, L. (2016). Finanzas 1: Contabilidad, planeación y administración financiera. IMCP. https://cutt.ly/wmOXxIb

Ogbebor, P., Osundina, O., \& Oyamendan, N. (2020). Internal Control and the Financial Performance of Selected Deposit Money Banks Listed in Nigeria. Developing Country Studies, 10(4), 61-73. https://doi.org/10.7176/dcs/10-4-07 
Pedraza, J., Ortiz, D., León, B., \& Naranjo, J. (2018). Importancia de los sistemas de control interno para pymes encaminado a la mitigación de riesgos operativos. Pensamiento Republicano, 9, 81-86. http://ojs.urepublicana.edu.co/index.php/pensamientorepublicano/article/view/543/451

Pelazas, M. (2015). Planificación de la auditoría. Ediciones Paraninfo S.A. https://cutt.ly/RnX5WPN

Pereira, C. (2019). Control interno en las empresas: Su aplicación y efectividad. Instituto Mexicano de Contadores Públicos, A.C. https://cutt.ly/8md1Nv1

Quinaluisa, N., Ponce. V., Muñoz, S., Ortega, X. \& Pérez, J. (2018). El control interno y sus herramientas de aplicación entre COSO y COCO. Cofin Habana, 12(1), 268-283. http://scielo.sld.cu/scielo.php?script=sci_arttext\&pid=S2073-60612018000100018\&lng=es\&tlng=es

Quispe, G., Arellano, O, \& Ayaviri, D. (2016). Aplicación de la Auditoría en las MyPEs del Ecuador: Un estudio de la demanda. Revista de investigación Altoandin, 18(4), 483 - 496. http://dx.doi.org/10.18271/ria.2016.241

Ruiz, Y., Valenzuela, V., \& Tapia, J. (2020). El control interno como instrumento de gestión de liquidez empresarial: un análisis meta-analítico en el cantón Latacunga. Universidad Ciencia y Tecnología, 24(106), 5-12. https://doi.org/10.47460/uct.v24i106.389

Saavedra, M., Tapia, B., \& Aguilar, M. (2016). La gestión financiera en las pymes del Distrito Federal, México. Revista Perspectiva Empresarial, 3(2), 55-69. https://doi.org/10.16967/rpe.v3n2a5

Saavedra, M. \& Camarena, M. (2018). La Gestión Financiera de las PYME en la Ciudad de México y su relación de la competitividad. Contaduría Universidad De Antioquia, (71), 149-173. https://revistas.udea.edu.co/index.php/cont/article/view/332828

Sanabria, F. (2021). Análisis del control interno en PYMES del Perú. Revista Multidisciplinaria Perspectivas Investigativas, 1(1), 9-13.

https://rperspectivasinvestigativas.org/ojs/index.php/multidiscipinaria/article/view/18

Sánchez, W. (2017). Teoría del control. Ediciones de la U. https://cutt.ly/EmfFJgC

Santiago, N. \& Gamboa, J. (2017). Gestión financiera empresarial. Consejo editorial de la Universidad Técnica de Ambato. https://cutt.ly/lnCQfTF

Santillana, J. (2013). Auditoría interna. Pearson Educación de México S.A. https://catedrafinancierags.files.wordpress.com/2012/04/auditoria-interna-juan-ramc3b3n-santillana.pdf

Sanusi, Z., Johari, R., Said, J., \& Iskandar, T. (2015). The Effects of Internal Control System, Financial Management and Accountability of NPOs: The Perspective of Mosques in Malaysia. Procedia Economics and Finance, 28 , 156-162. https://doi.org/10.1016/s2212-5671(15)01095-3

Setyaningsih, P. \& Nengzih, N. (2020). Internal Control, Organizational Culture, and Quality of Information Accounting to Prevent Fraud: Case Study from Indonesia's Agriculture Industry. International Journal of Financial Research, 11(4), 316-328. https://doi.org/10.5430/ijfr.v11n4p316

Serrano, P., Señalin, L., Vega, F., \& Herrera, J. (2018). El control interno como herramienta indispensable para una gestión financiera y contable eficiente en las empresas bananeras del cantón Machala (Ecuador). Espacios, 39(3), 30-43. https://www.revistaespacios.com/a18v39n03/a18v39n03p30.pdf

Sotomayor, A., Criollo, K. \& Gutiérrez, N. (2020). Control interno como herramienta eficiente para la gestión financiera y contable de las empresas camaroneras. 593 Digital Publisher CEIT, 5(6), 194-205. https://doi.org/10.33386/593dp.2020.6-1.387

Talaverano, D. \& Paima, R. (2018). Caracterización del control interno y la gestión de la Empresa Logística Peruana del Oriente S.A, Pucallpa, 2016. In Crescendo, 9(4), 649-665. https://cutt.ly/JnZIVa7

Tapia, C., Rueda, R. \& Silva, R. (2017). Auditoría Interna: Perspectivas de Vanguardia. Instituto Mexicano de Contadores Públicos. https://cutt.ly/CmfD0YM

Valderrama, S. (2011). Pasos para elaborar proyectos de investigación científica. Editorial San Marcos.

Valverde, M., Huachua, F., De los Santos, C., Solano, T., \& Vellón, V. (2017). Sistema de control interno en la gestión financiera de las micro y pequeñas empresas de la provincia de Barranca. Infinitum, 7(1), 65-69. https://doi.org/10.51431/infinitum.v7i1.63

Vega, L. y Nieves, A. (2016). Control de gestión y control interno: binomio indisoluble en la dirección. Espacios. 37 (12), 25-30. https://cutt.ly/inCjH4R

Vivanco, M. (2017). Los manuales de procedimientos como herramientas de control interno de una organización. Universidad y sociedad, 9(3), 247-252. http://scielo.sld.cu/pdf/rus/v9n3/rus38317.pdf 